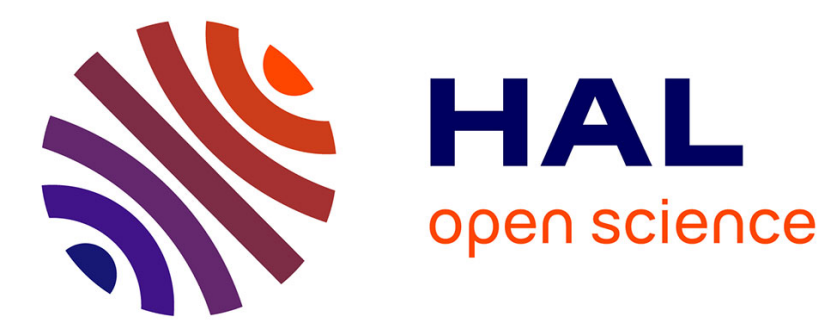

\title{
On Necessary and Sufficient Conditions for Fixed-Time Stability of Continuous Autonomous Systems
}

Francisco Lopez-Ramirez, Denis Efimov, Andrey Polyakov, Wilfrid

Perruquetti

\section{- To cite this version:}

Francisco Lopez-Ramirez, Denis Efimov, Andrey Polyakov, Wilfrid Perruquetti. On Necessary and Sufficient Conditions for Fixed-Time Stability of Continuous Autonomous Systems. Proc. 17th European Control Conference (ECC), Jun 2018, Limassol, Cyprus. hal-01755079

HAL Id: hal-01755079

https://hal.inria.fr/hal-01755079

Submitted on 30 Mar 2018

HAL is a multi-disciplinary open access archive for the deposit and dissemination of scientific research documents, whether they are published or not. The documents may come from teaching and research institutions in France or abroad, or from public or private research centers.
L'archive ouverte pluridisciplinaire HAL, est destinée au dépôt et à la diffusion de documents scientifiques de niveau recherche, publiés ou non, émanant des établissements d'enseignement et de recherche français ou étrangers, des laboratoires publics ou privés. 


\title{
On Necessary and Sufficient Conditions for Fixed-Time Stability of Continuous Autonomous Systems*
}

\author{
F. Lopez-Ramirez ${ }^{1}$, D. Efimov ${ }^{1,2}$, A. Polyakov ${ }^{1,2}$ and W. Perruquetti ${ }^{1,3}$
}

\begin{abstract}
Necessary and sufficient conditions for fixed-time stability of continuous autonomous system are given. Continuity of the settling-time function is considered and a characterization of fixed-time stability with continuous settling-time function is presented. Further refinements of these conditions that allow more constructive calculations are provided. Several academic examples are provided to illustrate the theoretical results.
\end{abstract}

\section{INTRODUCTION}

Lyapunov's direct method, a fundamental tool in control system theory, establishes that a dynamical system is asymptotically stable at the origin if there exists a smooth positive definite Lyapunov function with a negative definite derivative, derived for the system equations [1]. The converse counterpart of this result i.e. the proof that there exists a Lyapunov function for any system with an asymptotically stable equilibrium point, was later given by Kurzweil and Massera [1] in the 1950's and further strengthens the Lyapunov theoretical framework. This essential result has several extensions for stability of sets [2], stability of non-autonomous systems [1], differential inclusions [3], [4], input-to-state stability [5], etc., and converse results are often sought to complete the theoretical formulations. There are as well results that focus on the system's convergence rate that provide equivalent characterizations for exponential [6] or finite-time stability [7], [8], [9]. The latter refers to a type of stability where, contrarily to asymptotic stability, the trajectories of a given system converge exactly to zero in a finite amount of time [2], [7]. This type of stability is now well understood for continuous autonomous systems and necessary and sufficient conditions for it have been given in [9].

When all the trajectories of a finite-time stable system, within a specified domain, converge exactly to zero before a predefined time, i.e. known a priori, regardless of its initial conditions, we say that the system is fixed-time stable [10], [11], [12]. In practical applications where short convergence times are crucial, this powerful feature is highly desirable

*This work was partially supported by ANR 15 CE23 0007 (Project Finite4SoS), the Government of Russian Federation (Grant 074-U01) and the Ministry of Education and Science of Russian Federation (Project 14.Z50.31.0031).

${ }^{1}$ F. Lopez-Ramirez, D. Efimov, A. Polyakov and W. Perruquetti are with the Non-A Post Team, INRIA Lille-Nord Europe, 40, avenue Halley, Parc Scientifique de la Haute Borne, 59650, Villeneuve d'Ascq, France. email: \{francisco.lopez-ramirez, denis.efimov, andrey.polyakov, wilfrid.perruquetti\}einria.fr.

${ }^{2}$ D. Efimov and A. Polyakov are also with ITMO University, SaintPetersburg, Kronverski av. 49, Russia.

${ }^{3}$ W. Perruquetti is also with École Centrale de Lille and with CRIStAL Lab, UMR CNRS 9189, Cité Scientifique, 59651, Villneuve d'Ascq Cedex, France. since it provides an assurance on the convergence time even if the system's initial conditions are unknown. Since only sufficient conditions have been given to establish fixedtime stability [12], [13], the aim of the present work is to provide both necessary and sufficient ones as well as a more insightful characterization of this type of stability. The formal definitions of these notions and the problem statement are summarized in sections II and III. Section IV contains our main results. Subsection IV.A presents necessary and sufficient conditions for fixed-time stability. More constrictive sufficient conditions for the case of a continuous settling-time function are presented in Subsection IV.B and the corresponding converse results are developed in Subsection IV.C. The article closes with some conclusions and final remarks in Section V.

\section{NOTATION}

- $\mathbb{R}$ denotes the set of real numbers while $\mathbb{R}_{+}=\{x \in \mathbb{R}$ : $x>0\}$ and $\mathbb{R}_{\geq 0}=\{x \in \mathbb{R}: x \geq 0\}$.

- a class- $\mathcal{K}$ function is a continuous, strictly increasing function $\gamma: \mathbb{R}_{>0} \rightarrow \mathbb{R}_{>0}$ with $\gamma(0)=0$ while a class- $\mathcal{K}_{\infty}$ function is a class $\mathcal{K}$ function $\gamma$ such that $\gamma(s) \rightarrow \infty$ as $s \rightarrow \infty$.

- throughout the article, the domain $\mathcal{V} \subset \mathbb{R}^{n}$ will denote an open connected set containing the origin, such that $0 \in$ $\operatorname{int}(\mathcal{V})$, and $\partial \mathcal{V}$ will denote its boundary.

- a continuous function $V: \mathcal{V} \rightarrow \mathbb{R}_{\geq 0}$ is said to be radially unbounded on $\mathcal{V}$ if $V(x) \rightarrow+\infty$ as $x \rightarrow \partial \mathcal{V}$. If $\mathcal{V}$ is unbounded then, in addition, $V(x) \rightarrow+\infty$ as $\|x\| \rightarrow+\infty$.

- a function $V: \mathcal{V} \rightarrow \mathbb{R}$ is said to be of class- $\mathcal{C}^{1}$ if it is continuously differentiable. If the derivative $V^{(n)}(x)$ exists and it is continuous for all $x \in \mathcal{V}$ and for any integer $n$, then it is said to be $\mathcal{C}^{\infty}$.

- The brackets $\left\lceil\cdot J^{\nu}\right.$ simplify the notation $|\cdot|^{\nu} \operatorname{sign}(\cdot)$.

\section{PReliminaries}

Consider the following autonomous system

$$
\dot{x}=f(x), \quad x \in \mathcal{V},
$$

where $f: \mathcal{V} \rightarrow \mathbb{R}^{n}$ is a continuous function and $f(0)=0$. Let us assume that $f$ is such that (1) has the properties of existence and uniqueness of solutions in forward time. Then $\Phi(t, x)$ denotes the solution to system (1) starting from $x \in$ $\mathcal{V}$ at $t=0$.

Definition 1 ([1], [2], [12], [14]). The origin of the system (1) is said to be Lyapunov stable if for any $x_{0} \in \mathcal{V}$ the 
solution $\Phi\left(t, x_{0}\right)$ is defined for all $t \geq 0$, and for any $\epsilon>0$ there is $\delta>0$ such that for any $x_{0} \in \mathcal{V}$, if $\left\|x_{0}\right\| \leq \delta$ then $\left\|\Phi\left(t, x_{0}\right)\right\| \leq \epsilon$ for all $t \geq 0$

asymptotically stable if it is Lyapunov stable and $\left\|\Phi\left(t, x_{0}\right)\right\| \rightarrow 0$ as $t \rightarrow+\infty$ for any $x_{0} \in \mathcal{V}$;

finite-time stable if it is Lyapunov stable and finite-time converging from $\mathcal{V}$, i.e. for any $x_{0} \in \mathcal{V}$ there exists $0 \leq$ $T<+\infty$ such that $\Phi\left(t, x_{0}\right)=0$ for all $t \geq T$. The function $T\left(x_{0}\right)=\inf \left\{T \geq 0: \Phi\left(t, x_{0}\right)=0 \forall t \geq T\right\}$ is called the settling-time function of system (1);

uniformly finite-time stable if it is finite-time stable and the settling-time function $T\left(x_{0}\right)$ is locally bounded on $\mathcal{V}$;

fixed-time stable if it is uniformly finite-time stable and $\sup _{x_{0} \in \mathcal{V}} T\left(x_{0}\right)<+\infty$.

The set $\mathcal{V}$ is called the domain of attraction. If $\mathcal{V}=\mathbb{R}^{n}$ the corresponding stability becomes global.

The definition a finite-time and fixed-time stability involves the existence of a settling-time function, however, the definition doesn't address its continuity. Indeed, [7] provides a seemingly paradoxical example where the settling-time function of a finite-time stable systems tends to infinity as the initial conditions approach to the origin from a particular direction. Hence, the continuity of $T(x)$ on $x \in \mathcal{V}$ is a fundamental aspect to take into account in the study of finitetime stability.

\section{A. Lyapunov Functions and Lyapunov's Direct Method}

Definition 2 ([15]). A function $V: \mathcal{V} \rightarrow \mathbb{R}_{\geq 0}$ is called a strict Lyapunov function for system (1) if it fulfills the following properties:

L1 Positive Definiteness. $V(x)>0$ for all $x \in \mathcal{V} \backslash\{0\}$ and $V(0)=0$.

L2 $V$ is radially unbounded on $\mathcal{V}$.

L3 $V$ is of class $\mathcal{C}^{1}$ on $\mathcal{V}$ and

$$
\nabla V(x) \cdot f(x)<0 \text { for each } x \in \mathcal{V} \backslash\{0\} .
$$

Theorem 1 ([15, Theorems 5.2 and 5.3]). The origin of (1) is asymptotically stable on $\mathcal{V}$ if and only if there exists a strict Lyapunov function for (1).

This last theorem, also known as Lyapunov's direct method, establishes the structure of the Lyapunov methodology, that is, proposing a candidate positive definite function $V$ that satisfies a differential inequality. This same structure will remain throughout the theorems and corollaries to be presented, however, the right-hand side of $\mathbf{L 3}$ shall not only be negative definite, but it will involve a particular function of $V$.

The following corollary, used later in the paper, is a converse result of Lyapunov's direct method. What marks the difference from the converse statement of Theorem 1 is that instead of a differencial inequality, the condition on $\nabla V(x) \cdot f(x)$ involves a differential equality. The proof is omitted.

Corollary 1. Suppose that the origin of system (1) is asymptotically stable on $\mathcal{V}$. Then there exist a strict Lyapunov function $\tilde{V}: \mathcal{V} \rightarrow \mathbb{R}_{\geq 0}$ for (1), a continuous positive definite function $\tilde{W}: \mathcal{V} \rightarrow \mathbb{R}_{\geq 0}$ and two class- $\mathcal{K}_{\infty}$ functions $\alpha_{1}, \alpha_{2}$ that satisfy

$$
\begin{array}{ll}
\text { M1 } & \nabla \tilde{V}(x) \cdot f(x)=-\tilde{W}(x) \quad \forall x \in \mathcal{V} . \\
\text { M2 } & \alpha_{1}(\tilde{V}(x)) \leq \tilde{W}(x) \leq \alpha_{2}(\tilde{V}(x)) \quad \forall x \in \mathcal{V} .
\end{array}
$$

B. Relevant Theorems About Finite-Time Stability of Autonomous Systems

Given that fixed-time stability is in fact a particular type of finite-time stability, many of the results already obtained for the finite-time stable case remain valid for the fixed-time one. The following theorem gives necessary and sufficient conditions for finite-time stability of continuous autonomous systems, remark that here the settling-time function might well be discontinuous.

Theorem 2 ([9]). Let us consider system (1). The following properties are equivalent:

1) the origin of system (1) is finite-time stable on $\mathcal{V}$.

2) there exists a smooth strict Lyapunov function $V: \mathcal{V} \rightarrow$ $\mathbb{R}_{\geq 0}$ for system (1), satisfying for all $x \in \mathcal{V}$

$$
\int_{V(x)}^{0} \frac{d s}{\dot{V}\left(\Phi\left(\theta_{x}(s), x\right)\right)}<+\infty,
$$

where the map $s \stackrel{\theta_{x}}{\mapsto} t$ fulfills the identity $s=$ $V\left(\Phi\left(\theta_{x}(s), x\right)\right)$.

Moreover if 1) or 2) are verified, all smooth strict Lyapunov functions $V: \mathcal{V} \rightarrow \mathbb{R}_{\geq 0}$ for the system (1) satisfy for all $x \in \mathcal{V}$

$$
T(x)=\int_{V(x)}^{0} \frac{d s}{\dot{V}\left(\Phi\left(\theta_{x}(s), x\right)\right)}<+\infty .
$$

The following theorem, introduced initially in [7] provides a particular differential inequality from which finite-time stability with continuous settling-time function can be asserted.

Theorem 3 ([9], [7]). For the system (1), the following properties are equivalent:

1) the origin is finite-time stable on $\mathcal{V}$ with a Lipschitz continuous settling-time function.

2) there exist real numbers $c>0, \alpha \in(0,1)$ and a class$\mathcal{C}^{\infty}$ strict Lyapunov function $V: \mathcal{V} \rightarrow \mathbb{R}_{\geq 0}$ satisfying

$$
\dot{V}(x) \leq-c V(x)^{\alpha} \text { for all } x \in \mathcal{V} .
$$

An important result in finite-time stability theory, contained in the following lemma, states that for the settlingtime to be continuous in the whole domain of attraction, it suffices that $T$ is continuous at the origin.

Lemma 1 ([7, Proposition 2.4]). Suppose that the origin of system (1) is finite-time stable on $\mathcal{V}$. Let $T: \mathcal{V} \rightarrow \mathbb{R}_{\geq 0}$ be the settling-time function. Then the following statements hold:

i. If $x \in \mathcal{V}$ and $t \in \mathbb{R}_{\geq 0}$, then

$$
T(\Phi(t, x))=\max \{T(x)-t, 0\} .
$$

ii. $T$ is continuous on $\mathcal{V}$ if and only if $T$ is continuous at 0. 


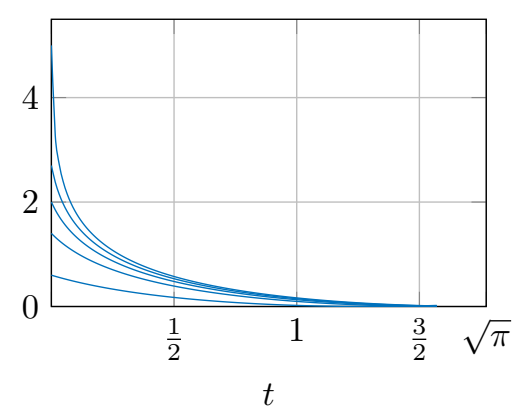

Fig. 1: Solutions of system (3) for five different initial conditions.

\section{MAin RESUlts}

Let us begin with the following motivating example of a fixed-time stable system.

Example 1. Consider the scalar system

$$
\dot{x}=-\lceil x\rfloor^{\frac{1}{2}} e^{|x|}, \quad x \in \mathbb{R},
$$

The trajectories of this dynamics, for any initial condition $x_{0} \in \mathbb{R}$ and any $t \geq 0$, can be obtained by direct integration and are given by

$\Phi\left(t, x_{0}\right)=\left\{\begin{array}{ll}\operatorname{erf}^{-1}\left[\operatorname{erf}\left(\left|x_{0}\right|^{\frac{1}{2}}\right)-\frac{t}{\sqrt{\pi}}\right]^{2} \operatorname{sign}\left(x_{0}\right), & \text { if } t \in\left[0, T\left(x_{0}\right)\right] \\ 0, & \text { if } t>T\left(x_{0}\right)\end{array}\right.$,

where $\operatorname{erf}(x)$ denotes the well-known error function $\operatorname{erf}(z):=$ $\frac{2}{\pi} \int_{0}^{z} e^{s^{2}} d s$. From the system's solutions, it is clear that for $t \geq \sqrt{\pi} \operatorname{erf}\left(\left|x_{0}\right|^{\frac{1}{2}}\right), \Phi\left(t, x_{0}\right)=0$ so that the settlingtime function is given by $T\left(x_{0}\right)=\sqrt{\pi} \operatorname{erf}\left(\left|x_{0}\right|^{\frac{1}{2}}\right)$. Moreover, this function is continuous and bounded on $\mathbb{R}$, indeed $\sup _{x_{0} \in \mathbb{R}} T\left(x_{0}\right)=\sqrt{\pi}$ so that all trajectories, regardless of where they start, converge exactly to zero in $t \leq \sqrt{\pi}$ (see Figure 1).

Our aim is to derive necessary and sufficient conditions for a dynamical system to behave as (3).

\section{A. Necessary and Sufficient Conditions For Fixed-Time Sta- bility}

Analogously to Theorem 2 we first present a necessary and sufficient condition for an autonomous system to be fixedtime stable, regardless of the continuity of the settling-time function.

Theorem 4. Consider system (1). Then the following properties are equivalent

1) The origin is fixed-time stable on $\mathcal{V}$.

2) There exists a strict Lyapunov function $V$ for system (1) satisfying for all $x \in \mathcal{V}$

$$
\int_{\sup _{x \in \mathcal{V}} V(x)}^{0} \frac{d s}{\dot{V}\left(\Phi\left(\theta_{x}(s), x\right)\right)}<+\infty,
$$

where $s \stackrel{\theta_{x}}{\mapsto} t$ is the inverse mapping of $t \mapsto V(\Phi(t, x))$.

Sketch of the proof. The proof is based on the fact that $\theta_{x}$ is a decreasing differentiable inverse mapping $(0, V(x)] \rightarrow$
$[0, T(x))$ that relates the strict Lyapunov function $V$ and the settling-time function $T$ by

$$
T(x)=\int_{0}^{T(x)} d t=\int_{V(x)}^{0} \theta_{x}^{\prime}(s) d s=\int_{V(x)}^{0} \frac{d s}{\dot{V}\left(\Phi\left(\theta_{x}(s), x\right)\right)} .
$$

Then 1) $\Leftrightarrow 2$ ) follows.

The condition 2) of this last theorem is in general difficult to verify. The following theorem, not only addresses the continuity of $T$, but also characterizes fixed-time stability by means of a strict Lyapunov function and a continuous positive definite function $r$. By means of this function, more constructive conditions can be obtained.

\section{B. Sufficient conditions for Fixed-Time Stability With Con-} tinuous Settling-Time Function

Theorem 5. Suppose that there exists a strict Lyapunov function $V: \mathcal{V} \rightarrow \mathbb{R}_{\geq 0}$ for system (1) such that

S1 there exists a continuous positive definite function

$$
r: \mathbb{R}_{\geq 0} \rightarrow \mathbb{R}_{\geq 0} \text { that verifies } \int_{0}^{\sup _{x \in \mathcal{V}} V(x)} \frac{d z}{r(z)}<+\infty
$$

S2 the inequality $\dot{V}(x) \leq-r(V(x))$ holds for all $x \in \mathcal{V}$.

Then the origin of (1) is fixed-time stable with continuous settling-time function $T: \mathcal{V} \rightarrow \mathbb{R}_{\geq 0}$ and

$$
T(x) \leq \int_{0}^{\sup _{x \in \mathcal{V}} V(x)} \frac{d z}{r(z)} \quad \forall x \in \mathcal{V} .
$$

Sketch of the proof. By exploiting the relation between $V$ and $T$ in (4) and the condition $\mathbf{S 2}$, it is possible to derive

$$
T(x) \leq \int_{0}^{V(x)} \frac{d s}{r(s)}
$$

and from condition S1 fixed-time stability follows. Taking any $x_{k}$ converging to zero, we have that $T\left(x_{k}\right) \leq$ $\int_{0}^{V\left(x_{k}\right)} \frac{d(s)}{r(s)}$. Since $V$ is continuous and $r(s)>0$ for all $s \in$ $\mathbb{R} \backslash\{0\}, \lim _{x_{k} \rightarrow 0} \int_{V\left(x_{k}\right)}^{0} \frac{d(s)}{r(s)}=0$, therefore $T$ is continuous at zero and by Lemma $1, T$ is continuous for all $x \in \mathcal{V}$.

The following corollary gives more insight about some of the particular forms that the characterizing function $r$ might take.

Corollary 2. Suppose there exists a Lyapunov function $V$ : $\mathbb{R}^{n} \rightarrow \mathbb{R}_{\geq 0}$ for system (1) such that

$$
\dot{V}(x) \leq-\left(a V(x)^{\alpha / \kappa}+b V(x)^{\beta / \kappa}\right)^{\kappa}, \quad x \in \mathbb{R}^{n},
$$

with $a, b, \alpha, \beta, \kappa>0, \kappa \alpha<1$ and $\kappa \beta>1$. Then the origin of (1) is fixed-time stable with continuous settling-time function $T(x) \leq 1 / a^{\kappa}(1-\kappa \alpha)+1 / b^{\kappa}(\kappa \beta-1)$.

In this Corollary, $r$ takes the particular form of $r(s)=$ $\left(a s^{\alpha / \kappa}+b s^{\beta / \kappa}\right)^{\kappa}$ and indeed satisfies conditions S1 and S2.

With the results provided so far let us present an example where we will determine fixed-time stability of a secondorder system and we will compare it with a finite-time one. 

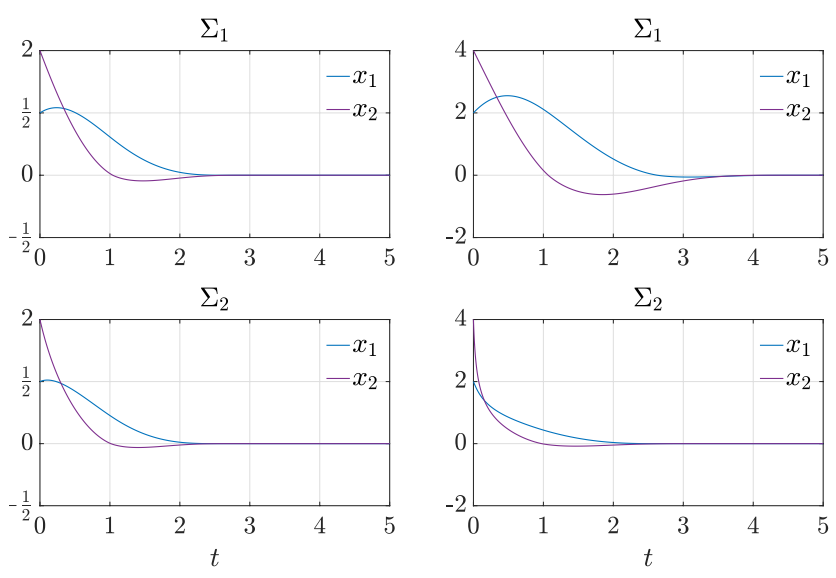

Fig. 2: Trajectories of $\Sigma_{1}$ and Fig. 3: Trajectories of $\Sigma_{1}$ and $\Sigma_{2}$ for $\gamma=2 / 3$ with initial $\Sigma_{2}$ for $\gamma=2 / 3$ with initial conditions $x_{0}=(1 / 2,1)$. conditions $x_{0}=(2,4)$.

Example 2. Consider the systems

$$
\begin{gathered}
\Sigma_{1}:\left\{\begin{array}{c}
\dot{x}_{1}=-\left\lceil x_{1}\right\rfloor^{\gamma}+x_{2} \\
\dot{x}_{2}=-\left\lceil x_{2}\right\rfloor^{\gamma}-x_{1}
\end{array}\right. \\
\Sigma_{2}:\left\{\begin{array}{c}
\dot{x}_{1}=-\left\lceil x_{1}\right\rfloor^{\gamma}-x_{1}^{3}+x_{2} \\
\dot{x}_{2}=-\left\lceil x_{2}\right\rfloor^{\gamma}-x_{2}^{3}-x_{1}
\end{array}\right.
\end{gathered}
$$

for $x \in \mathbb{R}^{2}, \gamma \in(0,1)$ and the Lyapunov function candidate $V(x)=\frac{1}{2}\left(x_{1}^{2}+x_{2}^{2}\right)$. For $\Sigma_{1}$ we have

$$
\left.\dot{V}(x)\right|_{\Sigma_{1}}=-\left(\left|x_{1}\right|^{\gamma+1}+\left|x_{2}\right|^{\gamma+1}\right) \leq-V(x)^{\frac{\gamma+1}{2}},
$$

while for $\Sigma_{2}$

$$
\begin{aligned}
\left.\dot{V}(x)\right|_{\Sigma_{2}} & =-\left(x_{1}^{4}+x_{2}^{4}\right)-\left(\left|x_{1}\right|^{\gamma+1}+\left|x_{2}\right|^{\gamma+1}\right) \\
& \leq-V(x)^{\frac{\gamma+1}{2}}-V(x)^{\gamma+1}
\end{aligned}
$$

where $\frac{\gamma+1}{2}<1$ and $1+\gamma>1$. Then, according to Corollary 2, $\Sigma_{1}$ is finite-time stable while $\Sigma_{2}$ is fixed-time stable with $T(x) \leq \frac{1}{1-\gamma}+\frac{1}{\gamma}$. Figure 2 shows the trajectories of $\Sigma_{1}, \Sigma_{2}$ under small initial conditions, notice that the settling time is similar in both systems. Figure 3 shows the trajectories of both systems for initial conditions slightly larger. Here it is possible to see how while the settling time of $\Sigma_{1}$ increases significantly, that of $\Sigma_{2}$ remains in a close vicinity.

\section{Necessary Conditions for Fixed-Time Stability With Con-} tinuous Settling-Time Function.

Theorem 6. Consider system (1) and suppose that the origin is fixed-time stable on $\mathcal{V}$ with a continuous settling-time function $T$. Then there exist a strict Lyapunov function $V$ and a class $-\mathcal{K}_{\infty}$ function $q$ that verify

$$
\begin{aligned}
& \text { N1 } \int_{0}^{\sup _{x \in \mathcal{V}} V(x)} \frac{d z}{q(z)}<+\infty ; \\
& \text { N2 }-q(V(x)) \leq \dot{V}(x) \quad \forall x \in \mathcal{V} .
\end{aligned}
$$

Sketch of the proof. From Corollary 1, we know that there exists some $q \in \mathcal{K}_{\infty}$ that verifies N1. Then, using (4) we obtain

$$
T(x) \geq \int_{0}^{V(x)} \frac{d s}{q(s)}
$$

and the result can be readily derived.

The following proposition is a converse result of Corollary 2 for $\kappa=1$.

Proposition 1. Suppose that the origin of (1) is fixed-time stable on $\mathbb{R}^{n}$ with a continuous settling-time function $T$. Then there exist a strict Lyapunov function $V$ and some constants $a, b>0, \alpha \in(0,1)$ and $\beta>1$ such that

$$
\begin{gathered}
\dot{V}(x) \leq-\left(a V(x)^{\alpha}+b V(x)^{\beta}\right) \forall x \in \mathbb{R}^{n} . \\
\text { V. Conclusions }
\end{gathered}
$$

For the first time, the necessary and sufficient conditions for fixed-time stability of continuous autonomous systems have been presented taking into account the continuity of the settling-time function and a characterization of this property by means of a pair of functions $(V, r)$ in the sufficiency case and $(V, q)$ in the necessary one has also been introduced. Further studies on the Hölder and/or Lipschitz continuity of the settling-time function might be interesting for future research topics as well as complete conditions for fixed-time controllability and stabilizability for the general nonlinear case.

\section{REFERENCES}

[1] H. K. Khalil, Nonlinear Systems, ser. NJ 07458. Upper Saddle River: Prentice-Hall, 1996.

[2] E. Roxin, "On finite stability in control systems," Rendiconti del Circolo Matematico di Palermo, vol. 15, pp. 273-283, 1966.

[3] A. F. Filippov, Differential equations with discontinuous righthand sides: control systems. Springer Science \&amp; Business Media, 2013, vol. 18.

[4] F. H. Clarke, Y. S. Ledyaev, R. J. Stern, and P. R. Wolenski, Nonsmooth analysis and control theory. Springer Science \&amp; Business Media, 2008, vol. 178.

[5] Y. Lin, E. D. Sontag, and Y. Wang, "A smooth converse Lyapunov theorem for robust stability," SIAM J. Control Optim., vol. 34, no. 1, pp. $124-160,1996$.

[6] S. F. Hafstein, "A constructive converse lyapunov theorem on exponential stability," Discrete and Continuous Dynamical Systems, vol. 10, no. 3, pp. 657-678, 2004.

[7] S. P. Bhat and D. S. Bernstein, "Finite time stability of continuous autonomous systems," SIAM J. Control Optim., vol. 38, no. 3, pp. 751-766, 2000.

[8] E. Moulay and W. Perruquetti, "Finite-time stability conditions for non-autonomous continuous systems," International Journal of Control, vol. 81(5), pp. 797-803, 2008.

[9] — - "Finite time stability and stabilization of a class of continuous systems," Journal of Mathematical Analysis and Application, vol. 323(2), pp. 1430-1443, 2006.

[10] V. Andrieu, L. Praly, and A. Astolfi, "Homogeneous Approximation, Recursive Observer Design, and Output Feedback," SIAM J. Control Optimization, vol. 47, no. 4, pp. 1814-1850, 2008.

[11] E. Cruz-Zavala, J. Moreno, and L. Fridman, "Uniform robust exact differentiator," IEEE Transactions on Automatic Control, vol. 56, no. 11, pp. 2727-2733, 2011.

[12] A. Polyakov, "Nonlinear feedback design for fixed-time stabilization of linear control systems," IEEE Transactions on Automatic Control, vol. 57, no. 8, pp. 2106-2110, 2012.

[13] A. Polyakov, D. Efimov, and W. Perruquetti, "Robust stabilization of MIMO systems in finite/fixed time," International Journal of Robust and Nonlinear Control, vol. 26, no. 1, pp. 69-90, 2016.

[14] A. Bacciotti and L. Rosier, Lyapunov Functions and Stability in Control Theory, 2nd ed., B. Springer, Ed. Springer, 2005.

[15] N. N. Krasovskij, Stability of motion: applications of Lyapunov's second method to differential systems and equations with delay. Stanford University Press, 1963. 